Research Article

\title{
SOX1 and PAX1 Are Hypermethylated in Cervical Adenocarcinoma and Associated with Better Prognosis
}

\author{
Zitong Zhao, Xiaoye Zhang, Xueheng Zhao, Jingting Cai, Na-Yi Yuan Wu $\mathbb{D}$, \\ and Jing Wang
}

Hunan Cancer Hospital, The Affiliated Cancer Hospital of Xiangya School of Medicine, Central South University, China

Correspondence should be addressed to Na-Yi Yuan Wu; wunayiyuan@163.com and Jing Wang; wangjing0081@hnca.org.cn

Zitong Zhao and Xiaoye Zhang contributed equally to this work.

Received 15 July 2020; Revised 24 November 2020; Accepted 27 November 2020; Published 8 December 2020

Academic Editor: Tao Chen

Copyright $\odot 2020$ Zitong Zhao et al. This is an open access article distributed under the Creative Commons Attribution License, which permits unrestricted use, distribution, and reproduction in any medium, provided the original work is properly cited.

\begin{abstract}
Background. The increased risk and poor survival outcome of cervical adenocarcinoma (CAC) demand for effective early diagnostic biomarkers that can predict the disease progression and outcome. The purpose of this study was to investigate the value of methylation status of SOX1 and PAX1 in the detection and prognosis of CAC. Methods. We performed a quantitative methylation-specific polymerase chain reaction in 205 cervical paraffin-embedded specimens (175 CACs, 30 noncancer cervical tissues). Overall and progression-free survival (OS and PFS, respectively) rates were calculated and compared using the KaplanMeier method. The prognostic value of $S O X 1^{m}$ and $P A X 1^{m}$ on CAC patients was assessed by the Cox regression model. A mathematical formula combining $S O X 1^{m}, P A X 1^{m}$, and age was constructed for survival prediction. Results. The methylation status of SOX1 and PAX1 was higher in CAC tissues than in noncancer cervical tissues. In addition, SOX $1^{m}$-positive CAC patients showed a higher 5 -year OS rate than $S O X 1^{m}$-negative patients. In CAC patients with smaller tumor size $(<4 \mathrm{~cm})$, the $P A X 1^{m}$-positive group showed a higher 5-year PFS rate than the $P A X 1^{m}$-negative group. In the algorithm combining $S O X 1^{m}, P A X 1^{m}$, and age, the low-risk group showed a better 5-year OS and PFS rate than the high-risk group. Conclusion. SOX1 and PAX1 methylation levels are higher in CAC than in normal cervical tissues and are potential biomarkers for monitoring CAC prognosis.
\end{abstract}

\section{Introduction}

Cervical cancer is the second commonest tumor in women worldwide [1]. Moreover, it is a major cause of cancerrelated death among women in developing countries [2]. Cervical adenocarcinoma (CAC) ranks second after cervical squamous cell carcinoma (SCC) as the most common pathological type. Although the use of human papillomavirus (HPV) vaccines and effective Pap smear screening have significantly decreased the incidence rate of cervical carcinoma in most regions, the percentage of CAC has been increasing, especially in younger women, accounting for $20-25 \%$ of all cervical carcinoma in some developed countries [2,3]. Moreover, the high propensity of CAC for ovarian metastases always leads to fertility destruction in young women [4-10]. Several studies have shown that at the same stage, CAC has a worse prognosis than SCC, mainly because of its higher rate of metastase [6] and lower sensitivity to radiotherapy and chemotherapy [11]. The increased frequency and poor survival outcome raise the need for useful biomarkers that can predict the progression and prognosis of CAC.

Epigenetic alterations include heritable DNA methylation and histone protein modifications that do not affect the DNA transcriptional sequence [12-15]. DNA methylation is an epigenetic alteration, which always occurs in the early stage of carcinogenesis, leading to lessen even lost expression of the methylated gene [16]. Expression of SOX1 correlated with early embryogenesis, central nervous system development, and neural stem cell maintenance [17]. Nonetheless, hypermethylation in the promoter region and/or somatic mutations in the so-called tumor suppressor genes might cause silencing or inhibition of SOX1, which in turn 
may result in cancer cell proliferation and migration and finally progression of cervical carcinogenesis [18]. Hypermethylated SOX1 $\left(S O X 1^{m}\right)$ has been associated with several cancer types, including hepatocellular cancer, lung cancer, urothelial bladder cancer, endometrial cancer, and SCC [19-22]. PAX1 expression is correlated with embryogenesis, particularly with the development of the thymus, parathyroid glands, and skeleton [23-25]. Hypermethylated PAX1 $\left(P A X 1^{m}\right)$ has been found in ovarian cancer, oral cancer, and SCC [26-29]. Our previous study confirmed SOX1 and $P A X 1$ methylation as promising screening biomarkers in cervical neoplasia, mainly in high-grade squamous intraepithelial lesions and SCC, because of its remarkable discriminating ability between normal tissues and high-grade cervical lesions [30-33]. However, CAC and SCC are different with respect to tumor development, progression, and molecular pathology. It remains unknown whether the methylation status of SOX1 and PAX1 is different between CAC and SCC. In addition, the methylation level and prognostic value of $S O X 1$ and $P A X 1$ for CAC are unclear. In this study, we investigated the methylation levels of SOX1 and PAX1 differ in CAC and evaluated the potential value of SOX1 and $P A X 1$ gene methylation for prognosis in CAC.

\section{Methods}

2.1. Patients and Tissue Specimens. A total of 205 cervical paraffin-embedded specimens between 2013 and 2015 were collected from the Hunan Cancer Hospital, the Affiliated Cancer Hospital of Xiangya School of Medicine, Central South University, including 30 noncancer cervical samples and 175 adenocarcinoma samples (Table 1). The patients' demographic and clinicopathological data, including clinical stage (FIGO Committee on Gynecologic Oncology, 2009), tumor size, depth of invasion, histologic tumor grade, lymph node metastasis, and treatment modality, were recorded. The study protocols were agreed by the Hunan Cancer Hospital ethics committee (project number: 2015[01]) and the Chinese Clinical Trial Registry (registration number: ChiCTR1800018931).

2.2. DNA Preparation, Bisulfite Conversion, and Quantitative Methylation-Specific Polymerase Chain Reaction (qMSP). An ISO17025-certified laboratory (iStat Biomedical Co., Ltd., New Taipei City, Taiwan) carried out total methylation tests. They first deparaffinized paraffin-embedded cervical tissues and then extracted genomic DNA (gDNA) samples and bisulfite converted by using an Epigene ${ }^{\mathrm{TM}}$ nucleic acid extraction kit and an Epigene ${ }^{\mathrm{TM}}$ bisulfite conversion kit (iStat Biomedical Co., Ltd., New Taipei City, Taiwan). Quantitative methylation-specific PCR (qMSP) was then used for analyzing the methylation level of $S O X 1$ and $P A X 1$ by the TaqMan Probe system in a Light Cycler LC480 system (Roche Applied Science, Penzberg, Germany). Our previous study described specific primers and probes for qMSP [31, 34, 35]. The registered A375 and CaSki two cancer cell lines were treated as methylation and nonmethylation controls to ensure the quality of the bisulfite conversion and qMSP processes. The DNA methylation level was assessed as the
TABle 1: Clinicopathologic characteristics of 205 patients.

\begin{tabular}{lc}
\hline Characteristics & Numbers $(\%)$ \\
\hline Specimens $(N=205)$ & $175(85.37 \%)$ \\
Adenocarcinoma & $30(14.63 \%)$ \\
Noncancer tissues & \\
Cancer group $(N=175)$ & \\
Age (years) & $96(54.86 \%)$ \\
$\quad<50$ & $79(45.14 \%)$ \\
$\geq 50$ & \\
FIGO stage & $127(71.57 \%)$ \\
$\quad<$ IIB & $48(27.43 \%)$ \\
$\geq$ IIB & \\
Tumor size & $101(57.71 \%)$ \\
$<4$ & $72(41.14 \%)$ \\
$\geq 4$ & \\
Deep of invasion & $74(42.29 \%)$ \\
$<1 / 2$ & $90(51.43 \%)$ \\
$\geq 1 / 2$ & \\
LNM & $126(72 \%)$ \\
No & $41(23.43 \%)$ \\
Yes & \\
Histologic grade & $119(68 \%)$ \\
Well/moderate & $45(25.71 \%)$ \\
\hline Poor & $G y+c 009 y$ \\
\hline
\end{tabular}

Abbreviations: FIGO: International Federation of Gynecology and Obstetrics; LNM: lymph node metastasis.

methylation index (M-index) using the formula [36]: $10,000 \times 2^{\text {(Cpvalueofgene-CpvalueofCOL2A) }}$. SOX1 and PAX1 (positive) were deemed to be hypermethylated (positive) if the delta $\mathrm{Cp}$ was smaller than 11 and 9, respectively.

2.3. Statistical Analyses. The cut-off values for $S O X 1^{m}$ and $P A X 1^{m}$ were generated from 205 clinical samples (175 CACs and 30 noncancer cervical tissues). Receiver operating characteristic (ROC) curves were performed, and the area under the ROC curve (AUC) was calculated for the detection of the CAC.

All statistical analyses were performed using GraphPad Prism $^{\circledR} 7.00$ (GraphPad, La Jolla, CA, USA) and SPSS Statistics 24 (SPSS, Inc., Chicago, IL, USA). The correlation between $S O X 1^{m}$ or $P A X 1^{m}$ and each clinicopathological characteristic of the CAC patients was evaluated by the Mann-Whitney and Dunnett's tests. Kaplan-Meier method was used to describe the progression-free survival and overall survival (PFS and OS). The PFS was judged from treatment to the date of the first relapse at any site or death including all causes, and OS was calculated from treatment to death covering any cause. Hazard ratio (HRs) was calculated with multivariate Cox regression analysis.

2.4. A Mathematical Algorithm Combining $S O X 1^{m}, P A X 1^{m}$, and Age for CAC Prognosis Prediction. To investigate the effectiveness of the combination of the methylation statuses of these two genes and the clinicopathological factors to 


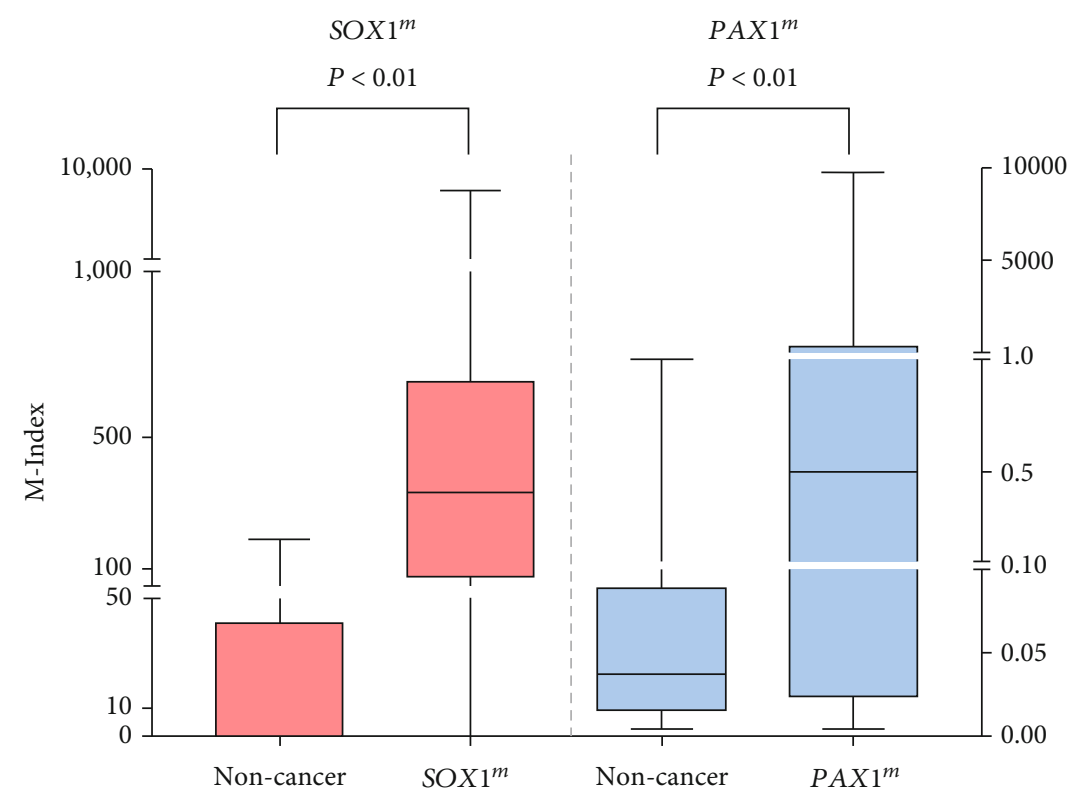

(a)
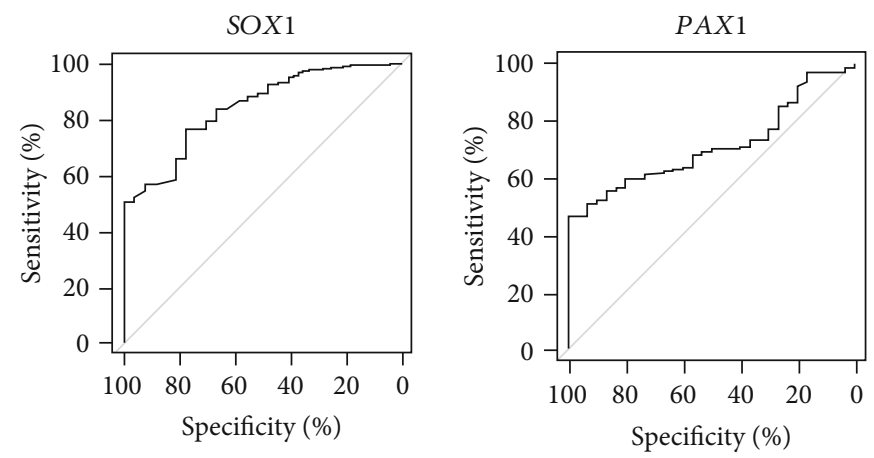

\begin{tabular}{cccccc}
\multicolumn{5}{c}{ Adenocarcinoma detection } \\
\hline Gene $^{m}$ & Cut of value & Sensitivity & Specificity & AUC & $P$ \\
\hline SOX1 & $\Delta$ Cp: 11 & $87.22 \%$ & $56.67 \%$ & $88.42 \%$ & $<0.001$ \\
PAX1 $^{m}$ & $\Delta$ Cp: 9 & $44.30 \%$ & $100 \%$ & $70.80 \%$ & $<0.01$ \\
\hline
\end{tabular}

(b)

FIGURE 1: SOX1 and PAX1 methylation levels in cervical adenocarcinoma. (a) Methylation-Index (M-Index) of SOX1 and PAX1 methylation between the noncancer group (30 noncancer tissues) and cervical cancer group (175 adenocarcinoma), $p<0.01$; (b) the area under the ROC curve for the SOX1 and PAX1 methylation assay was calculated for exploring cervical cancer. The sensitivity and specificity of SOX1 methylation were $87.22 \%$ and $56.67 \%$, respectively, with a cut-off point of $\Delta \mathrm{Cp}=11$. The AUC was $88.42 \%$. And the sensitivity and specificity of PAX1 methylation were $40.30 \%$ and $100 \%$, separately, with a cut-off point of $\triangle \mathrm{Cp}=9$. The AUC was $70.80 \%$.

predict the clinical outcome, we constructed a mathematical formula for survival prediction. Each patient was assigned with a risk score in accordance with a linear combination of the expression level of the two genes. The risk score was calculated as follows: risk score $=\left[W 1 \times S O X 1^{m}\right]+[W 2$ $\left.\times P A X 1^{m}\right]+[W 3 \times$ age $]$. The weight factors $(W 1-3)$ were generated from the regression coefficients derived from the aforementioned-univariate Cox regression analysis (Lossos et al., 2004). We divided patients into low-risk and highrisk groups according to the median risk score as the cut- off point. The OS and PFS were then estimated by the Kaplan-Meier method.

\section{Results}

3.1. SOX1 and PAX1 Are Hypermethylated in CAC Tissues than in Noncancer Cervical Tissues. Among our 205 cervical specimens, 30 were noncancer cervical samples and 175 were adenocarcinoma samples (Table 1). The mean M-index for SOX1 (476.80 \pm 92.47 and $0.48 \pm 0.29$, respectively, $p<0.05)$ 
TABLE 2: Association between SOX1m or PAX1m and clinicopathological characteristics in 175 CAC patients.

\begin{tabular}{|c|c|c|c|c|c|c|c|}
\hline Variable & $\begin{array}{l}\text { Number of patients } \\
\qquad(n=175)\end{array}$ & Positive & $\begin{array}{l}S O X 1^{m} \\
\text { Negative }\end{array}$ & $p$ value & Positive & $\begin{array}{l}P A X 1^{m} \\
\text { Negative }\end{array}$ & $p$ value \\
\hline Age (years) & & & & 0.625 & & & 0.91 \\
\hline$<50$ & $96(54.86 \%)$ & 83 & 13 & & 28 & 68 & \\
\hline$\geq 50$ & $79(45.14 \%)$ & 70 & 9 & & & & \\
\hline FIGO stage & & & & 0.937 & & & 0.766 \\
\hline$<\mathrm{IIB}$ & $127(71.57 \%)$ & 112 & 15 & & 36 & 91 & \\
\hline$\geq \mathrm{IIB}$ & $48(27.43 \%)$ & 41 & 6 & & 14 & 33 & \\
\hline Tumor size & & & & 0.748 & & & 0.412 \\
\hline$<4$ & $101(57.71 \%)$ & 89 & 12 & & 27 & 74 & \\
\hline$\geq 4$ & $72(41.14 \%)$ & 62 & 10 & & 23 & 49 & \\
\hline Deep of invasion & & & & 0.929 & & & 0.863 \\
\hline$<1 / 2$ & $74(42.29 \%)$ & 64 & 10 & & 21 & 53 & \\
\hline$\geq 1 / 2$ & $90(51.43 \%)$ & 78 & 12 & & 24 & 66 & \\
\hline LNM & & & & 0.418 & & & 0.17 \\
\hline No & $126(72 \%)$ & 111 & 15 & & 37 & 89 & \\
\hline Yes & $41(23.43 \%)$ & 34 & 7 & & 8 & 33 & \\
\hline Histologic grade & & & & 0.644 & & & 0.146 \\
\hline Well/moderate & $119(68 \%)$ & 104 & 15 & & 33 & 86 & \\
\hline Poor & $45(25.71 \%)$ & 38 & 7 & & 17 & 28 & \\
\hline
\end{tabular}

and PAX1 (515.70 \pm 56.30 and $28.19 \pm 9.19$, respectively, $p<0.05)$ was significantly higher in CAC tissues than in noncancer tissues (Figure 1(a)). By ROC analysis, the positive cut-off value for $S O X 1^{m}$ was $\Delta \mathrm{Cp} \leq 11$, with a high AUC level of $88.42 \%$, a sensitivity of $87.22 \%$, and specificity of $56.67 \%$. The positive cut-off value for $P A X 1^{m}$ was $\triangle \mathrm{Cp} \leq 9$, with a high AUC level of $70.80 \%$, sensitivity of $44.30 \%$, and specificity of $100 \%$ (Figure 1(b)), which suggested that $S O X 1^{m}$ and $P A X 1^{m}$ may be detection biomarkers for CAC.

The SOX1 and PAX1 methylation statuses showed no significant difference based on age, FIGO stage, tumor size, depth of invasion, lymph node metastasis, and histologic grade in CAC patients (Table 2).

3.2. Hypermethylated SOX1 and PAX1 Are Associated with Better Survival in CAC Patients. Further studies were conducted to investigate whether the methylation of SOX1 and $P A X 1$ was correlated with the prognosis in CAC patients. The SOX $1^{m}$-positive group showed a higher 5-year OS rate of $93.35 \%$ than the $S O X 1^{m}$-negative group, which showed a 5 -year OS rate of $68.29 \%(p=0.048)$ (Figure $2(a))$. While no remarkable finding was obtained in the analysis of the 5-year PFS rate. For PAX1, there was no evident difference in 5-year OS rate or 5-year PFS rate between these two groups (data not shown). However, in CAC patients with smaller tumor size $(<4 \mathrm{~cm})$, the $P A X 1^{m}$-positive group had a higher 5-year PFS rate than the $P A X 1^{m}$-negative group (100\% vs. $82.4 \%, p=0.044$ ) (Figure $2(\mathrm{~b})$ ).

3.3. Algorithm Combining $S O X 1^{m}, P A X 1^{m}$, and Age for Prognosis of CAC Patients. We established an algorithm for quantifying age, $S O X 1^{m}$, and $P A X 1^{m}$ as a prognostic factor to calculate the recurrence risk and outcome of patients with
CAC. The final algorithm for OS was as follows: risk score $=\left[-1.109 \times S O X 1^{m}\right]+\left[-0.849 \times P A X 1^{m}\right]+[0.399 \times$ age $]$. The final algorithm for PFS was as follows: risk score $=$ $\left[-0.586 \times S O X 1^{m}\right]+\left[-0.553 \times P A X 1^{m}\right]+[0.674 \times$ age $]$. The algorithm divided patients into low-risk and high-risk groups. However, the low-risk group showed a better 5-year OS rate $(95.89 \%$ vs. $81.47 \%, p=0.019)$ (Figure $3(\mathrm{a})$ ) and a much better 5 -year PFS rate $(90.58 \%$ vs. $72.50 \%, p=0.006)$ than the high-risk group (Figure 3(b)).

\section{Discussion}

It is well known that the prognosis of CAC is worse than cervical SCC, even for early-stage patients, especially in developing countries [37]. Gene methylation is an epigenetic modification, which has tumor-suppressive or tumorigenic two opposite effects, possibly playing key roles in carcinogenesis and cancer progression. Before this study, the methylation status and prognostic value of $S O X 1^{m}$ and $P A X 1^{m}$ for CAC were unclear. In this study, we observed higher methylation levels of SOX1 and PAX1 in CAC than in noncancer cervical tissues. For the detection of CAC, SOX $1^{m}$ showed $87.22 \%$ sensitivity and $56.67 \%$ specificity, while $P A X 1^{m}$ showed $44.30 \%$ sensitivity and $100 \%$ specificity. Moreover, several studies have demonstrated that $P A X 1$ methylation increases following increased disease grade: $P A X 1$ methylation in SCC > high - grade squamous intraepithelial lesion (HSIL) > low - grade squamous intraepithelial lesion (LSIL) $>$ normal tissue $[38,39]$. These suggest that hypermethylation of $S O X 1$ and $P A X 1$ might play an important role in the diagnosis and cancer progression of CAC.

The current study also showed, for the first time, that CAC patients who are $S O X 1^{m}$-positive show a better 

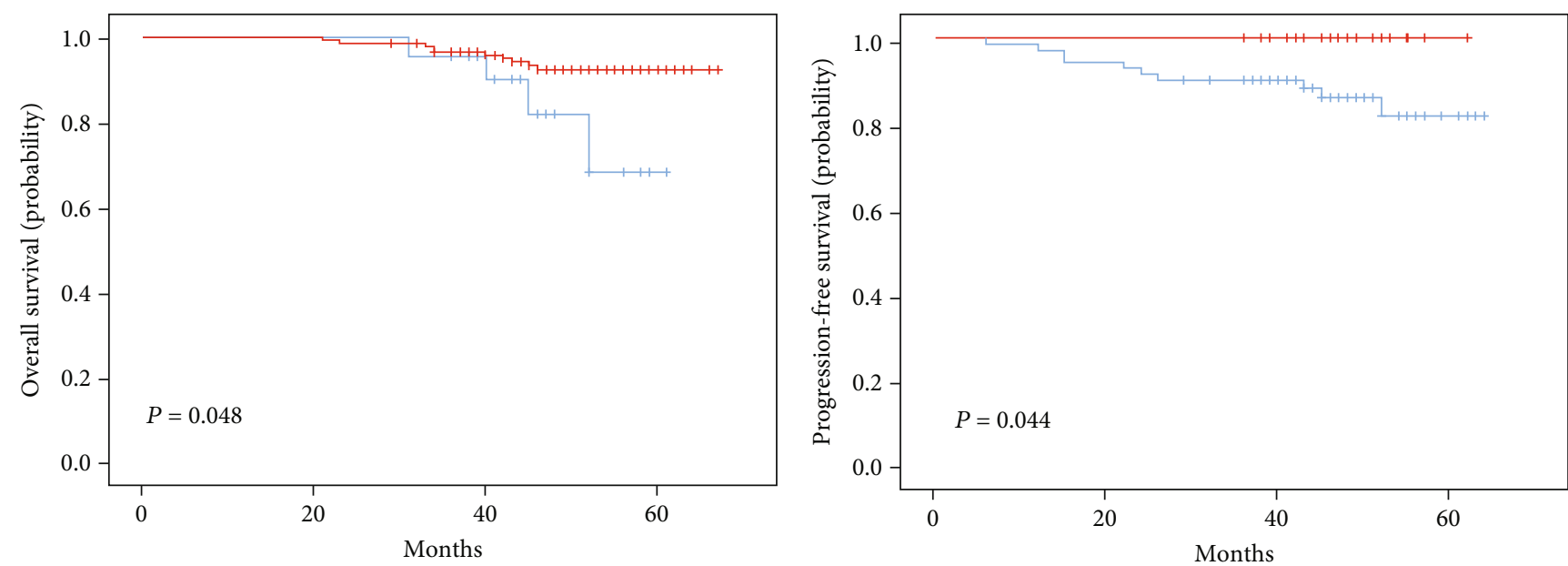

\begin{tabular}{|c|c|c|}
\hline & $N$ & 5-year overall survival rate \\
\hline$S O X 1^{m+}$ & 153 & $93.35 \%$ \\
\hline$S O X 1^{m-}$ & 22 & $68.29 \%$ \\
\hline
\end{tabular}

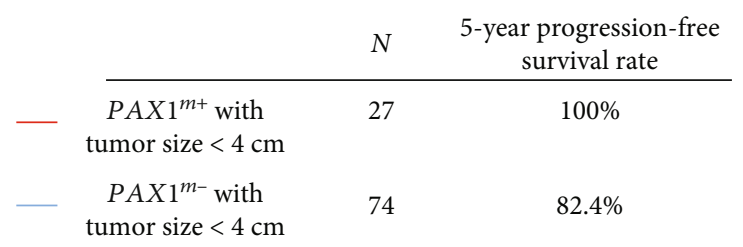

(a)

(b)

FIgure 2: Association of $S O X 1$ and $P A X 1$ methylation status with CAC patients' survival. (a) $S O X 1^{m}$-positive patients have a longer OS rate than SOX1 $1^{m}$-negative patients $(93.35 \%$ vs. $68.29 \%, p=0.048)$; (b) in CAC patients with smaller tumor size $(<4 \mathrm{~cm}), P A X 1^{m}$-positive group had a higher 5-year PFS rate than the $P A X 1^{m}$-negative group ( $100 \%$ vs. $\left.82.4 \%, p=0.044\right)$.

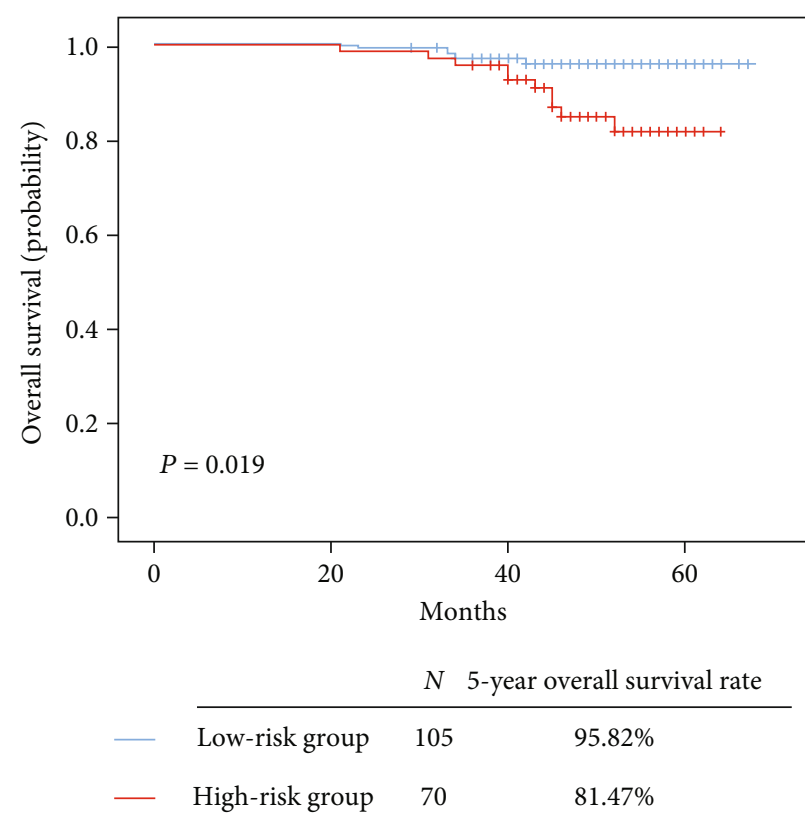

(a)

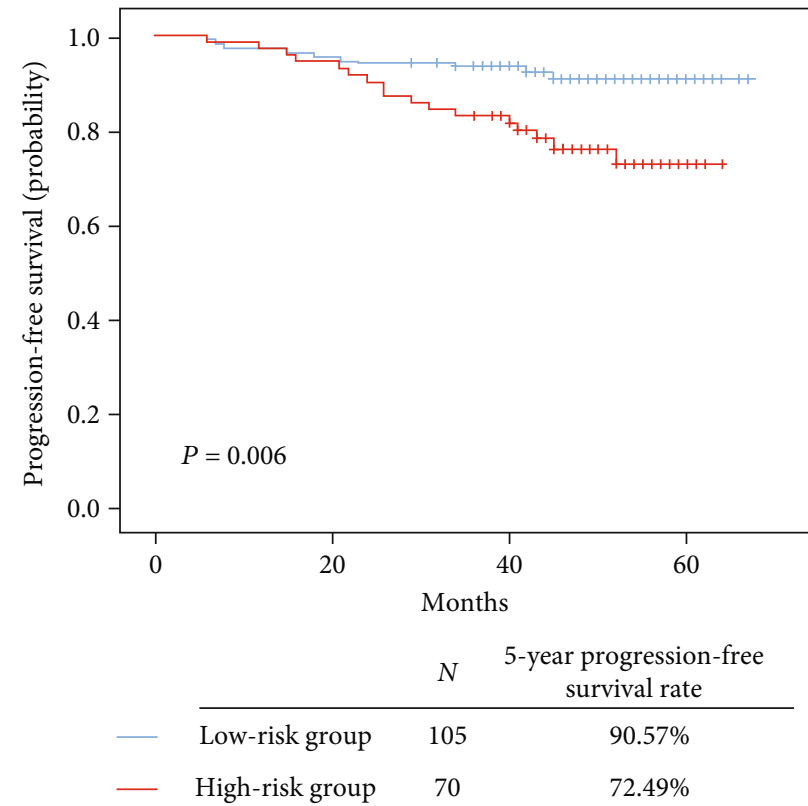

(b)

FIGURE 3: Algorithm combining clinicopathologic factor and genetic test results. The algorithm divided patients into high-risk and low-risk groups. The low-risk group showed a better 5 -year overall survival (OS) rate (95.89\% vs. 81.47\%, $p=0.019)$ (a) and better 5-year progressionfree survival (PFS) rate $(90.58 \%$ vs. $72.50 \%, p=0.006)$ than the high-risk group (b).

prognosis, suggesting that SOX1 gene methylation has the potential to predict the 5-year OS. In CAC patients with small tumor size $(<4 \mathrm{~cm}), P A X 1^{m}$-positive patients showed a longer 5-year PFS, suggesting that $P A X 1$ gene methylation might be useful for monitoring the 5-year progression in CAC patients with small tumor size. This study, meanwhile, 
has several limitations. First, our patients all came from a single medical center and the sample size was comparatively small. Second, the detailed molecular relationship between $S O X 1^{m}$ and $P A X 1^{m}$ in CAC has not been explored, which deserves further investigation.

Despite the poor prognosis of CAC, there is still no valid prognostic risk model. By far, age is a strong risk factor for cancer $[40,41]$. The current paper showed corresponding directed changes in DNA methylation with age, which is characterized by hypermethylation of targets of polycomb group proteins (PCGTs) that are crucial in embryonic stem cell lineage differentiation [42]. In the algorithm combining $S O X 1^{m}, P A X 1^{m}$, and age, the low-risk group, composed of high methylation level of SOX1 and PAX1 and younger age, showed a significantly higher 5-year OS rate and 5-year PFS rate than the high-risk group. These results suggested that the algorithm has the potential for a 5-year CAC prognosis.

In our previous study, negative gene methylation correlated with high protein expression, which increased the resistance of cervical cancer cells to radiation and chemotherapy [43]. In further studies, it will be essential to analyze the correlation between $S O X 1$ and PAX1 methylation status and sensitivity of the cervical cancer cell to radiotherapy and chemotherapy.

In conclusion, this study suggests that SOX1 and PAX1 methylation levels are higher in CAC than in cervical SCC, and $S O X 1^{m}$ and $P A X 1^{m}$ are potential biomarkers for monitoring CAC prognosis.

\section{Abbreviations \\ CAC: Cervical adenocarcinoma \\ SCC: Cervical squamous cell carcinoma \\ SOX $1^{\mathrm{m}}$ : Methylation status of SOX1 \\ $\mathrm{PAX}_{1}{ }^{\mathrm{m}}$ : Methylation status of PAX1 \\ FIGO: International Federation of Gynecology and Obstetrics \\ PFS: Progression-free survival \\ OS: $\quad$ Overall survival.}

\section{Data Availability}

The data used to support the findings of this study are included in the article.

\section{Ethical Approval}

This project was licensed by the Hunan Cancer Hospital ethics committee (project number: 2015[01]) and the Chinese Clinical Trial Registry (registration number: ChiCTR1800018931).

\section{Conflicts of Interest}

The authors declare that they have no competing interests.

\section{Authors' Contributions}

J Wang, NY Wu, and ZT Zhao did the concept and design; ZT Zhao did the literature search; NY Wu and ZT Zhao did the clinical studies; XH Zhao, ZT Zhao, and NY Wu did the data acquisition; ZT Zhao and NY Wu did the data analysis; ZT Zhao and NY Wu did the statistical analysis; ZT Zhao, XY Zhang and NY Wu did the manuscript preparation; all authors did the manuscript editing and manuscript review. Zitong Zhao and Xiaoye Zhang contributed equally to this work.

\section{Acknowledgments}

The authors would like to thank all members of the Jing Wang laboratory, iStat Biomedical Co., Ltd., and Hunan Hongya Gene Technology Co., Ltd. This work was supported by the Clinical Research Center in Gynecologic Cancer, Hunan Cancer Hospital, National Natural Science Foundation of China (82003050 and 81972836), Natural Science Foundation of Hunan Province (2020JJ5338 and 2020JJ8020), the Foundation from Social development science and technology division (2018SK2121, kq2004138, and kq1801104), Scientific research project of Hunan Health Commission (20201487), and Hunan Provincial Health Commission (20201487).

\section{References}

[1] A. Jemal, F. Bray, M. M. Center, J. Ferlay, E. Ward, and D. Forman, "Global cancer statistics," CA: a cancer journal for clinicians, vol. 65, no. 2, pp. 87-108, 2015.

[2] L. T. Gien, M. C. Beauchemin, and G. Thomas, "Adenocarcinoma: a unique cervical cancer," Gynecologic oncology, vol. 116, no. 1, pp. 140-146, 2010.

[3] J. van der Horst, A. G. Siebers, J. Bulten, L. F. Massuger, and I. M. de Kok, "Increasing incidence of invasive and in situ cervical adenocarcinoma in the Netherlands during 2004-2013," Cancer Medicine, vol. 6, no. 2, pp. 416-423, 2017.

[4] C. W. Drescher, M. P. Hopkins, and J. A. Roberts, "Comparison of the pattern of metastatic spread of squamous cell cancer and adenocarcinoma of the uterine cervix," Gynecologic oncology, vol. 33, no. 3, pp. 340-343, 1989.

[5] P. J. Eifel, M. Morris, M. J. Oswald, J. Taylor Wharton, and L. Delclos, "Adenocarcinoma of the uterine cervix. Prognosis and patterns of failure in 367 cases," Cancer, vol. 65, no. 11, pp. 2507-2514, 1990.

[6] P. J. Eifel, T. W. Burke, M. Morris, and T. L. Smith, "Adenocarcinoma as an independent risk factor for disease recurrence in patients with stage IB cervical carcinoma," Gynecologic oncology, vol. 59, no. 1, pp. 38-44, 1995.

[7] T. Irie, J. Kigawa, Y. Minagawa et al., "Prognosis and clinicopathological characteristics of Ib-IIb adenocarcinoma of the uterine cervix in patients who have had radical hysterectomy," European journal of surgical oncology, vol. 26, no. 5, pp. 464467, 2000.

[8] M. Shimada, J. Kigawa, R. Nishimura et al., "Ovarian metastasis in carcinoma of the uterine cervix," Gynecologic oncology, vol. 101, no. 2, pp. 234-237, 2006.

[9] F. Landoni, V. Zanagnolo, L. Lovato-Diaz et al., "Ovarian metastases in early-stage cervical cancer (IA2-IIA): a multicenter retrospective study of 1965 patients (a Cooperative Task Force study)," International Journal of Gynecological Cancer, vol. 17, no. 3, pp. 623-628, 2007. 
[10] X. B. Jiao, J. Hu, and L. R. Zhu, "The safety of ovarian preservation in early-stage adenocarcinoma compared with squamous cell carcinoma of uterine cervix: a systematic review and meta-analysis of observational studies," International Journal of Gynecologic Cancer, vol. 26, no. 8, pp. 1510-1514, 2016.

[11] S. Takeuchi, "Biology and treatment of cervical adenocarcinoma," Chinese Journal of Cancer Research, vol. 28, no. 2, pp. 254-262, 2016.

[12] P. W. Laird, "The power and the promise of DNA methylation markers," Nature Reviews Cancer, vol. 3, no. 4, pp. 253-266, 2003.

[13] S. Sharma, T. K. Kelly, and P. A. J. J. Carcinogenesis, "Epigenetics in Cancer," Carcinogenesis, vol. 31, no. 1, pp. 27-36, 2013.

[14] A. P. Feinberg and B. Vogelstein, "A technique for radiolabeling DNA restriction endonuclease fragments to high specific activity," Analytical biochemistry, vol. 132, no. 1, pp. 6-13, 1984.

[15] P. A. Jones and S. B. Baylin, "The fundamental role of epigenetic events in cancer," Nature Reviews Genetics, vol. 3, no. 6, pp. 415-428, 2002.

[16] J. Martinez-Galan, J. R. Delgado, R. D. Ávila et al., “544 DNA methylation: an epigenetic pathway to cancer and a promising target for anticancer therapy in breast cancer," European Journal of Cancer Supplements, vol. 8, no. 7, p. 173, 2010.

[17] L. Kan, N. Israsena, Z. Zhang et al., "Sox1 acts through multiple independent pathways to promote neurogenesis," Developmental biology, vol. 269, no. 2, pp. 580-594, 2004.

[18] Y. W. Lin, C. M. Tsao, P. N. Yu, Y. L. Shih, C. H. Lin, and M. D. Yan, "SOX1 suppresses cell growth and invasion in cervical cancer," Gynecologic oncology, vol. 131, no. 1, pp. 174-181, 2013.

[19] X. Y. Liu, Y. C. Fan, S. Gao et al., "Methylation of SOX1 and VIM promoters in serum as potential biomarkers for hepatocellular carcinoma," Neoplasma, vol. 64, no. 5, p. 745, 2017.

[20] L. Ning, X. Li, S. Li, S. Zhou, Q. J. B. Zhou, and B. R. Communications, "Cisplatin-induced downregulation of SOX1 increases drug resistance by activating autophagy in nonsmall cell lung cancer cell," Biochemical and biophysical research communications, vol. 439, no. 2, pp. 187-190, 2013.

[21] J. I. López, J. C. Angulo, A. Martín et al., “A DNA hypermethylation profile reveals new potential biomarkers for the evaluation of prognosis in urothelial bladder cancer," Apmis, vol. 125, no. 9, pp. 787-796, 2017.

[22] H. C. Lai, Y. C. Wang, M. H. Yu et al., "DNA methylation as a biomarker for the detection of hidden carcinoma in endometrial atypical hyperplasia," Gynecologic oncology, vol. 135, no. 3, pp. 552-559, 2014.

[23] J. M. Mcgaughran, A. Oates, D. Donnai, A. P. Read, and M. Tassabehji, "Mutations in PAX1 may be associated with Klippel-Feil syndrome," European Journal of Human Genetics, vol. 11, no. 6, p. 468, 2003.

[24] J. Wallin, H. Eibel, A. Neubüser, J. Wilting, H. Koseki, and R. Balling, "Pax1 is expressed during development of the thymus epithelium and is required for normal T-cell maturation," Development, vol. 122, no. 1, pp. 23-30, 1996.

[25] S. Schnittger, V. V. Rao, U. Deutsch, P. Gruss, R. Balling, and I. Hansmann, "Pax1, a member of the paired box-containing class of developmental control genes, is mapped to human chromosome 20p11.2 by in situ hybridization (ISH and FISH)," Genomics, vol. 14, no. 3, p. 740, 1992.

[26] R. Guerrero-Preston, C. Michailidi, L. Marchionni et al., "Key tumor suppressor genes inactivated by "greater promoter" methylation and somatic mutations in head and neck cancer," Epigenetics, vol. 9, no. 7, pp. 1031-1046, 2014.

[27] D. Lang, S. K. Powell, R. S. Plummer, K. P. Young, and B. A. Ruggeri, "PAX genes: roles in development, pathophysiology, and cancer," Biochemical pharmacology, vol. 73, no. 1, pp. 114, 2007.

[28] Y. Y. Kan, Y. L. Liou, H. J. Wang et al., "PAX1 methylation as a potential biomarker for cervical cancer screening," International journal of cancer, vol. 24, no. 5, pp. 928934, 2014.

[29] H. Y. Su, H. C. Lai, Y. W. Lin, Y. C. Chou, C. Y. Liu, and M. H. $\mathrm{Yu}$, "An epigenetic marker panel for screening and prognostic prediction of ovarian cancer," International Journal of Cancer, vol. 124, no. 2, pp. 387-393, 2009.

[30] Y. Y. Kan, Y. L. Liou, H. J. Wang et al., "PAX1 methylation as a potential biomarker for cervical cancer screening," International Journal of Gynecologic Cancer, vol. 24, no. 5, p. 928, 2014.

[31] H. C. Lai, Y. C. Ou, T. C. Chen et al., "PAX1/SOX1?DNA methylation and cervical neoplasia detection: a Taiwanese Gynecologic Oncology Group (TGOG) study," Cancer medicine, vol. 3, no. 4, pp. 1062-1074, 2014.

[32] C. Tai-Kuang, K. Feng-Yi, L. Yu-Ping, W. Hui-Chen, Y. Cheng-Ping, and L. J. D. C. Hung-Cheng, "Triage of cervical cytological diagnoses of atypical squamous cells by DNA methylation of paired boxed gene 1 (PAX1)," Diagnostic Cytopathology, vol. 41, no. 1, pp. 41-46, 2013.

[33] H. C. Lai, Y. W. Lin, R. L. Huang et al., "Quantitative DNA methylation analysis detects cervical intraepithelial neoplasms type 3 and worse," Cancer, vol. 116, no. 18, pp. 4266-4274, 2010.

[34] Y. L. Liou, Y. Zhang, Y. Liu et al., "Comparison of HPV genotyping and methylated ZNF582 as triage for women with equivocal liquid-based cytology results," Clinical Epigenetics, vol. 7, no. 1, p. 50, 2015.

[35] Y. L. Liou, T. L. Zhang, T. Yan et al., "Combined clinical and genetic testing algorithm for cervical cancer diagnosis," Clinical epigenetics, vol. 8, no. 1, p. 66, 2016.

[36] R. L. Huang, C. C. Chang, P. H. Su et al., "Methylomic analysis identifies frequent DNA methylation of zinc finger protein 582 (ZNF582) in cervical neoplasms," PLoS One, vol. 7, no. 7, article e41060, 2012.

[37] N. T. Lua, N. D. Chinh, N. T. Hue et al., "Survey-based cancer mortality in the Lao PDR, 2007-08," The Asian Pacific Journal of Cancer Prevention, vol. 12, no. 10, pp. 2495-2498, 2011.

[38] E. H. Lim, S. L. Ng, J. L. Li et al., "Cervical dysplasia: assessing methylation status (Methylight) of CCNA1, DAPK1, HS3ST2, PAX1 and TFPI2 to improve diagnostic accuracy," Gynecologic oncology, vol. 119, no. 2, pp. 225-231, 2010.

[39] H. C. Lai, Y. W. Lin, T. H. Huang et al., "Identification of novel DNA methylation markers in cervical cancer," International journal of cancer, vol. 123, no. 1, pp. 161-167, 2008.

[40] M. C. White, D. M. Holman, J. E. Boehm, L. A. Peipins, M. Grossman, and S. J. Henley, "Age and cancer risk: a potentially modifiable relationship," American journal of preventive medicine, vol. 46, pp. S7-15, 2014. 
[41] Group UCSW, "United States cancer statistics: 1999-2005 incidence and mortality web-based report," in US Department of Health and Human Services, Centers for Disease Control and Prevention and National Cancer Institute, US DHHS/CDC/NIH-NCI, Atlanta, 2009.

[42] A. E. Teschendorff, U. Menon, A. Gentry-Maharaj et al., "Agedependent DNA methylation of genes that are suppressed in stem celels is a hallmark of cancer," Genome research, vol. 20, no. 4, pp. 440-446, 2010.

[43] N. Y. Wu, X. Zhang, T. Chu et al., "High methylation of ZNF582 in cervical adenocarcinoma affects radiosensitivity and prognosis," Annals of Translational Medicine, vol. 7, 14 pages, 2019. 